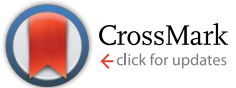

Cite this: RSC Adv., 2016, 6, 94294

Received 22nd July 2016

Accepted 27th September 2016

DOI: $10.1039 / c 6 r a 18692 k$

www.rsc.org/advances

\section{Microheterogeneity in binary mixtures of aliphatic alcohols and alkanes: ATR-IR/NIR spectroscopic and chemometric studies}

\author{
Władysław Wrzeszcz, Paweł Tomza, Michał Kwaśniewicz, Sylwester Mazurek \\ and Mirosław Antoni Czarnecki*
}

\begin{abstract}
Aliphatic alcohols form homogeneous mixtures with alkanes of similar size and structure, however at a molecular level one can expect the presence of both the homo and heteroclusters leading to the local heterogeneity. Recently, we observed this phenomenon in the binary mixtures of methanol with aliphatic alcohols [RSC Adv., 2016, 6, 37195]. This paper provides new and comprehensive information on the structure of alcohol/alkane mixtures at a molecular level. Besides, we studied the relationship between the chain structure and the deviation from the ideal mixture. A particular attention was paid for the difference between the linear and cyclic alcohols and alkanes. For studies we selected two alcohols: 1hexanol, cyclohexanol, and two alkanes: $n$-hexane, cyclohexane. By combining these two pairs of compounds, we obtained four different alcohol/alkane mixtures. The inhomogeneity distribution of molecules in these mixtures and deviation from the ideality was characterized by ATR-IR/NIR excess absorption spectra and chemometric methods. Obtained results allow us to conclude that the separation at a molecular level and the extent of deviation from the ideality depends on the degree of association of the alcohol and the similarity of alkyl parts. As 1-hexanol is more associated than cyclohexanol, 1hexanol/n-hexane mixture is the closest to the ideal mixture. In contrast, cyclohexanol/n-hexane is the most non-ideal mixture since in this case the smaller degree of self-association of cyclohexanol is coupled with different structure of alcohol and alkane chains.
\end{abstract}

\section{Introduction}

At a macroscopic level, long chain aliphatic alcohols form homogeneous mixtures with alkanes of similar size and structure. Though the literature on self-association of alcohols in nonpolar solvents is very rich it does not present a uniform picture. $^{1-14}$ Fletcher and Heller postulated the presence of monomers as well as the linear and cyclic tetramers of 1-octanol and 1-butanol in $n$-decane solutions but they did not observe the dimers. ${ }^{1}$ In contrast, Asprion et al. assumed that most of alcohols in $n$-hexane exist in equilibrium between the monomers, dimers and oligomers (mostly pentamers). ${ }^{2}$ Kunst $e t$ al. reported that association of $n$-alcohols in alkanes is well fitted by monomer-dimer-tetramer model. ${ }^{3}$ The authors claim that the length of alkyl chain $(n>2)$ has no appreciable effect on creation of the linear dimers and cyclic tetramers. Based on results of NIR and NMR studies of 1-octanol in $n$-decane Iwahashi et al. suggested that the cyclic tetramer is the main associated species for $n$-alcohols in rod-like alkane solvents. ${ }^{4} \mathrm{On}$ the other hand, Car-Parrinello molecular dynamics calculations by Stubbs and Siepmann reveal that the distribution of

Faculty of Chemistry, University of Wroctaw, F. Joliot-Curie 14, 50-383 Wrockaw, Poland. E-mail: miroslaw.czarnecki@chem.uni.wroc.pl; Fax: +48-71-3282348 alcohol associates in nonpolar solvents is more complex than the frequently assumed equilibrium of monomers and cyclic tetramers. ${ }^{5}$ The authors also suggest that there is no simple correlation between the $\mathrm{OH}$ peak shift and size or structure of the associates. As shown, the cooperativity effect appears to be responsible for differentiation of the polymer-like (cooperative) and dimer-like (noncooperative) peaks in the vibrational spectrum. The frequency shift for the 'dimer-like' band is around $100 \mathrm{~cm}^{-1}$ with respect to the $\mathrm{OH}$ monomer, while the 'polymerlike' band is usually shifted more than $200 \mathrm{~cm}^{-1}$ in alcohol/ alkane mixtures. The cyclic associates are expected to absorb in the 'polymer-like' region. Gupta and Brinkley also emphasized importance of the hydrogen bonding cooperativity in 1-alkanol/ $n$-alkane mixtures. ${ }^{6}$ They suggested that the equilibrium constant for the second hydrogen bond is 10 times higher than that for the first hydrogen bond. In contrast, Solms et al. reported that FT-IR spectra of different 1-alkanol/n-alkane mixtures can be precisely modeled by SAFT method without taking into account the hydrogen bonding cooperativity. ${ }^{7}$

Wilson et al. have shown that the degree of association in alcohols dissolved in long chain alkanes appears to be independent of the extent of van der Waals or hydrophobic interactions that might exist in these solutions. ${ }^{8}$ It depends mainly on the alcohol concentration, but is independent of both the 
alcohol and the alkane chain length. On the contrary, NIR spectroscopic study coupled with 2D correlation analysis and chemometric methods demonstrated an opposite behavior., Comparison of results for various aliphatic alcohols reveals that the strength of hydrogen bonding weakens with an increase in both the alcohol order and the chain length. Dielectric relaxation studies of mixtures of aliphatic alcohols with $n$-alkanes reveals that the structure of the liquid phase is dependent on the relative chain length of the alcohol and the alkane. ${ }^{11}$ When the alcohol chain is longer than that of the alkane, the alkane is mainly solved in the hydrocarbon region of the alcohol clusters. In the other case, in the mixture microheterogenity exists with the separate clusters of the alkane. Flores et al. studied 1-hexanol association in cyclohexane by using NMR and NIR spectroscopies. ${ }^{12}$ The authors concluded that the structure of the mixture depends on its composition. At low 1-hexanol mole fraction $(X<0.05)$ dominates dispersion interactions and the molecules of alcohols exist mainly as monomers. When $X$ still further increases up to 0.2 in the mixture dominates the hydrogen bonding leading to creation of higher associates. In the range of 1-hexanol mole fraction from 0.2 to 0.9 the structure of the mixture is determined by dipolar interactions. Max and Chapados examined methanol in $n$-hexane by using ATR-IR spectroscopy. ${ }^{13}$ They suggested that the structure of this mixture depends on the methanol content and is well represented by two kinds of micelles. At high methanol content $\left(\mathrm{X}_{\mathrm{CH}_{3} \mathrm{OH}}>0.75\right)$ are created the micelles, while inverse micelles (the $\mathrm{OH}$ groups in the core) are formed in the low methanol concentration $\left(X_{\mathrm{CH}_{3} \mathrm{OH}}<0.25\right)$. In the intermediate concentrations $(0.25<X<$ $0.75)$ these two kinds of micelles compete giving rise to a phase separation. The authors also concluded that the hydrogen bonding interaction through the $\mathrm{OH}$ groups is much stronger than that of van der Waals interactions of the alkane chains.

When two compounds with different chain length or structure are mixed together, one can expect heterogeneity at a molecular level, despite of the macroscopic miscibility. The local heterogeneity simply results from the presence of different homo and heteroclusters. This phenomenon leads to anomalous behavior of physicochemical properties of the mixtures and is observed as a deviation from the ideality. It is of particular note that the separation at a molecular level was observed even in the methanol/ethanol mixture. ${ }^{15}$ Mello et al. suggested the presence of separate methanol and ethanol clusters without hydrogen bonding between different alcohol species. Recently, we reported microheterogeneity in binary mixtures of methanol with short chain aliphatic alcohols. ${ }^{16}$ As shown, in the entire range of compositions in the mixtures coexist the clusters of pure alcohols and the mixed clusters with an average mole ratio of $1: 1$. All mixtures deviate from the ideality and the largest deviation appears at equimolar mixture. At this composition about $50 \%$ of molecules are involved in the mixed clusters. These studies demonstrated that the degree of non-ideality in binary mixtures of methyl alcohol with the short chain aliphatic alcohols depends on the chain length and the order of the alcohol.

Most of previous studies on alcohol/alkane mixtures were performed at relatively low alcohol concentrations $(M<0.1)$ and were focused mainly on behavior of the alcohol. On the other hand, very little attention was paid for behavior of the aliphatic solvent. This work provides new and comprehensive information on the state of both alcohol and alkane in the mixture. The relationship between the extent of deviation from the ideality of alcohol/alkane mixtures and molecular structure of both components was elucidated. To realize this purpose we studied four mixtures: 1-hexanol $/ n$-hexane, 1-hexanol/cyclohexane, cyclohexanol $/ n$-hexane and cyclohexanol/cyclohexane by using ATR-IR/NIR spectroscopy combined with the excess absorption spectra and chemometric methods.

\section{Experimental details}

For studies we selected two alcohols: 1-hexanol (Merck, Germany), cyclohexanol (Eurochem BGD, Poland) and two alkanes: $n$-hexane (J. T. Baker, the Netherlands), cyclohexane (Carl Roth, Germany). All samples (purity $>99 \%$ ) were distilled and dried under freshly activated molecular sieves (4A). ATR-IR and NIR spectra were recorded at a resolution of $2 \mathrm{~cm}^{-1}$ and $4 \mathrm{~cm}^{-1}(256$ scans), respectively, on Nicolet Magna 860 spectrometer with DTGS detector. ATR-IR spectra were recorded with multireflection ZnSe crystal (PIKE), while NIR spectra were measured in a variable temperature quartz cell of $5 \mathrm{~mm}$ thickness (Hellma). The spectra of 1-hexanol $/ n$-hexane and 1-hexanol/ cyclohexane mixtures were recorded at $25^{\circ} \mathrm{C}$, while the spectra of cyclohexanol $/ n$-hexane and cyclohexanol/cyclohexane mixtures were recorded at $30^{\circ} \mathrm{C}$ (melting point of cyclohexanol is $\approx 26-27^{\circ} \mathrm{C}$ ) in the entire range of mole fractions with a step of $0.04 \pm 0.0001$. The mixtures were automatically prepared and dosed to ATR-IR and NIR cells by a flow-system constructed in our laboratory (Fig. 1). This system was controlled by portable computer and assured a high precision and repeatability of sample preparation. As a result, the experimental data were more accurate and provided more reliable information on small intensity variations.

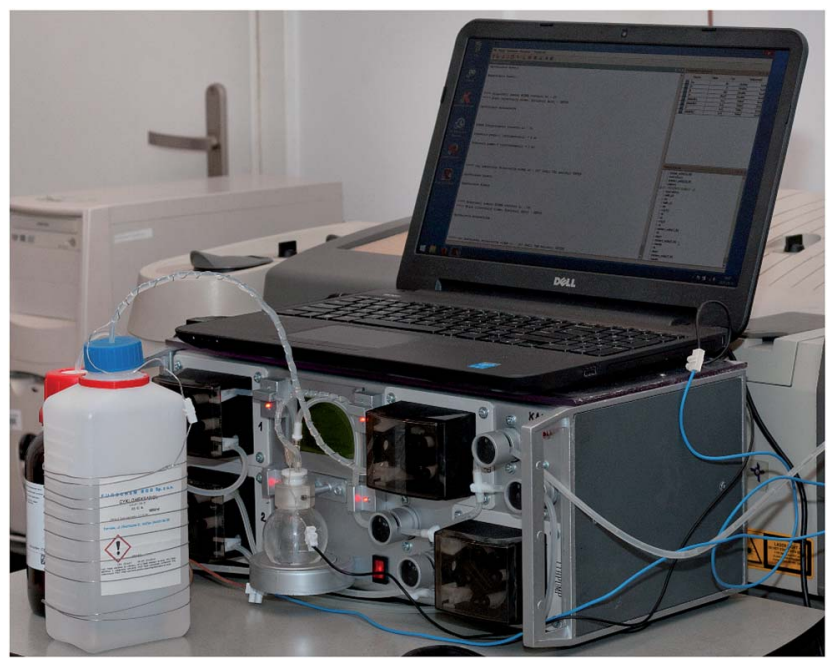

Fig. 1 Flow-system for automatic preparing and dosing samples to ATR-IR/NIR cells. 


\section{Computational details}

The baseline fluctuations in ATR-IR and NIR spectra were reduced by an offset at $4000 \mathrm{~cm}^{-1}$ and $9000 \mathrm{~cm}^{-1}$, respectively. The spectra have high signal-to-noise-ratio, and no other corrections were necessary. The number of components in the data matrix and the initial estimates of the concentration profiles for each component were obtained from principal component analysis (PCA) and evolving factor analysis (EFA). ${ }^{17,18}$ In addition, PCA was used for identification of the outliers. The actual concentration and spectral profiles of pure components were resolved by multivariate curve resolutionalternating least squares (MCR-ALS) method with constraints (non-negativity on concentrations and spectra, closure, equality). ${ }^{19,20}$ The chemometric analysis was achieved by PLSToolbox 8.2 (Eigenvector Research Inc.) for use with Matlab 9.0 (MathWorks).

The excess MIR/NIR absorption spectra $\left(A_{\text {exc }}\right)$ were calculated as a difference between the real spectra $(A)$ and the linear combination of the MIR/NIR spectra of neat alcohol $\left(A_{1}\right)$ and alkane $\left(A_{2}\right)$ :

$$
A_{\mathrm{exc}}=A-\left[A_{1} X_{1}+A_{2}\left(1-X_{1}\right)\right]
$$

where $X_{1}$ is the mole fraction of $A_{1}$. The part in the square brackets represents the spectra of the ideal mixture. Hence, the excess absorption spectrum is related to deviation of the
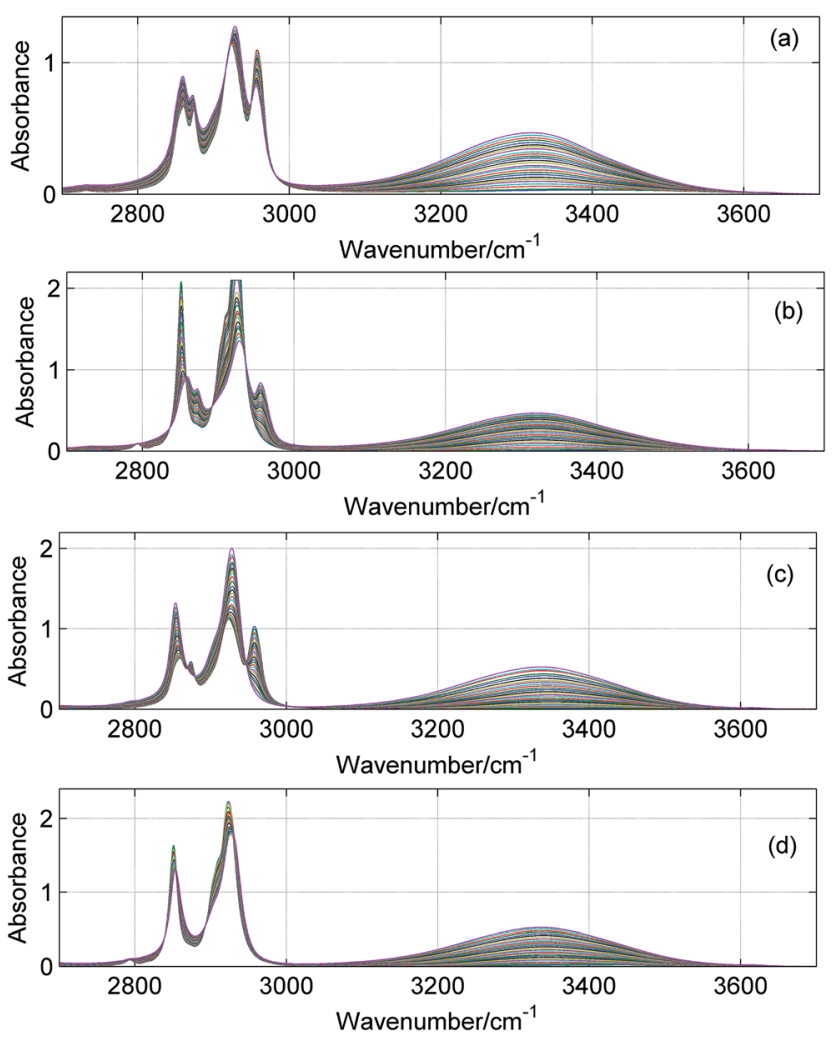

Fig. 2 ATR-IR spectra of 1-hexanol/n-hexane (a), 1-hexanol/cyclohexane (b), cyclohexanol/n-hexane (c) and cyclohexanol/cyclohexane (d) in the entire range of mole fractions with a step of 0.04 . mixture from the ideality. ${ }^{21,22}$ From the excess spectra one can calculate the values of ER, which allow for quantitative estimation of the deviation from the ideal mixture and comparison between different mixtures. This value was introduced in our previous paper ${ }^{\mathbf{1 6}}$ and is defined as below:

$$
\mathrm{ER}=\frac{\int\left|\operatorname{mean}\left(A_{\mathrm{exc}}\left(X_{\mathrm{alc}}\right)\right)\right|}{\int \operatorname{mean}\left(A\left(X_{\mathrm{alc}}\right)\right)} \times 100 \%
$$

where 'mean' stands for the composition-mean MIR/NIR excess absorption spectrum $\left(A_{\text {exc }}\right)$ or normal spectrum $(A)$ and $X_{\text {alc }}$ is the mole fraction of alcohol.

\section{Results and discussion}

\section{ATR-IR/NIR spectra}

ATR-IR and NIR spectra were recorded from 1000 to 12000 $\mathrm{cm}^{-1}$ in the entire range of mole fractions, but for analysis we selected the most important regions including the $\mathrm{CH}$ and $\mathrm{OH}$ stretching vibrations. In the $2800-3000 \mathrm{~cm}^{-1}$ range absorb the $\mathrm{C}-\mathrm{H}$ stretching vibrations of the methyl and methylene groups (Fig. 2), while the corresponding second overtones absorb from 8000 to $8700 \mathrm{~cm}^{-1}$ (Fig. 3). The broad band centered near 3300 $\mathrm{cm}^{-1}$ is due to the stretching vibration of the hydrogen-bonded $\mathrm{O}-\mathrm{H}$ group (Fig. 2), whereas its first overtone appears in the $6000-6900 \mathrm{~cm}^{-1}$ range (Fig. 3). It is of note that the band due to the free $\mathrm{OH}$ group does not occur in the MIR spectra. In contrast, this band clearly appears in the NIR spectra near 7100
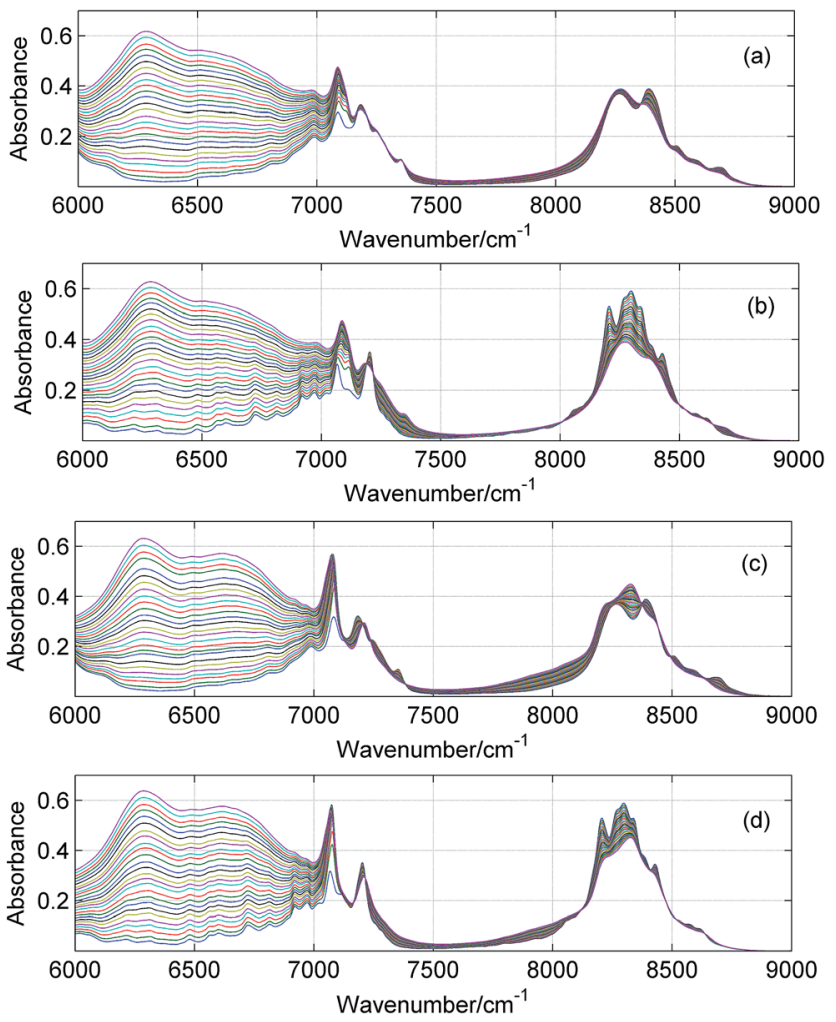

Fig. 3 NIR spectra of 1-hexanol/n-hexane (a), 1-hexanol/cyclohexane (b), cyclohexane/n-hexane (c) and cyclohexanol/cyclohexane (d) in the entire range of mole fractions with a step of 0.04 . 

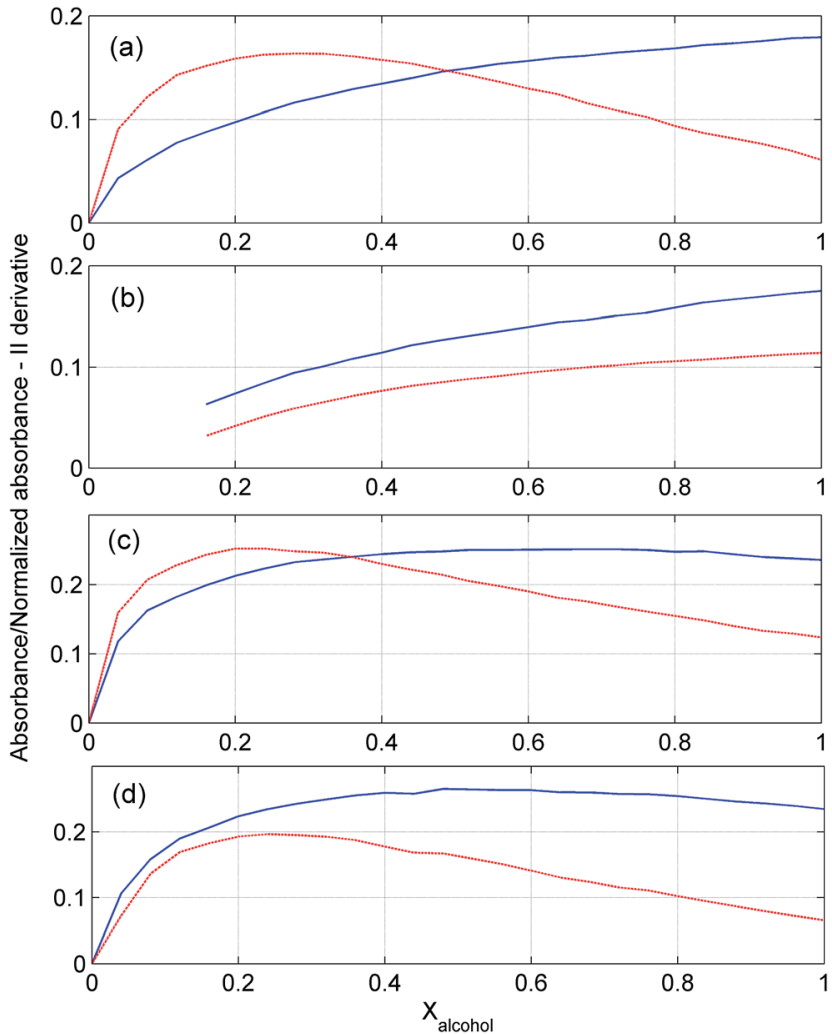

Fig. 4 Absorbance (solid, blue) and normalized second derivative (dashed, red) of the first overtone of the free $\mathrm{OH}$ for 1-hexanol/nhexane (a), 1-hexanol/cyclohexane (b), cyclohexanol/n-hexane (c) and cyclohexanol/cyclohexane (d) mixtures.

$\mathrm{cm}^{-1}$ (Fig. 3). Luck and Ditter have shown that vibrations of weak hydrogen bonds and free $\mathrm{OH}$ have weak fundamentals and strong overtones. ${ }^{23}$ The intensities of the bands assigned to the bonded $\mathrm{OH}$ groups increase with the increase in mole fraction of the alcohol. On the other hand, the spectral changes for the $\mathrm{CH}$ bands are more complex and depend on the chain structure of alcohol and alkane. Also the intensity changes for the band due to the free $\mathrm{OH}$ are not monotonic and follow more complex pattern (Fig. 4). Since this band is overlapped by the $\mathrm{CH}$ combination bands absorbing from 6900 to $7400 \mathrm{~cm}^{-1},{ }^{24}$ its spectral changes are better seen in the second derivative spectrum. In most of mixtures the maximum of these changes occurs at $X_{\text {alc }} \approx 0.2-0.3$. For small alcohol content the population of the free $\mathrm{OH}$ rapidly increases with $X_{\text {alc }}$. When the alcohol content still further increases, a part of alcohol molecules form hydrogen bonding and therefore this increase becomes slower. Finally, when $X_{\text {alc }}>0.3$ the population of the bonded $\mathrm{OH}$ increases faster as compared with an increase in the alcohol content. As a result, the overall population of the free $\mathrm{OH}$ decreases.

\section{ATR-IR/NIR excess absorption spectra}

Fig. 5 shows the composition-mean excess absorption spectra in MIR and NIR ranges. The values of ER calculated from these spectra are collected in Table 1. As can be seen from Fig. 5 and
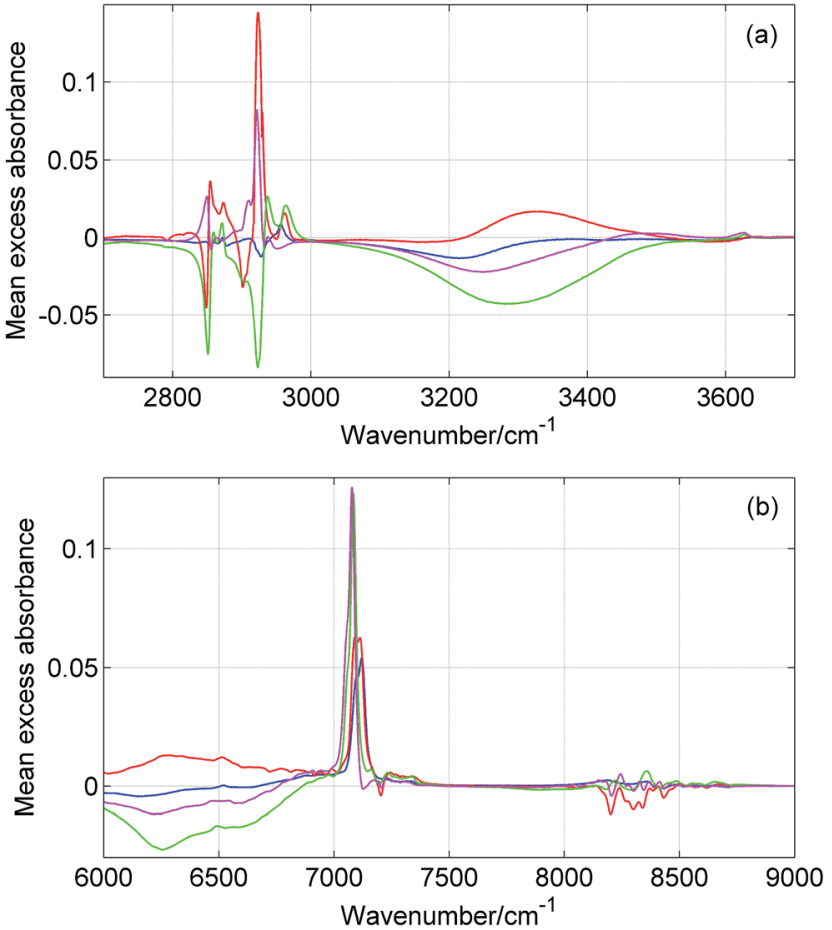

Fig. 5 Composition-mean excess MIR (a) and NIR (b) absorption spectra of 1-hexanol/n-hexane (blue), 1-hexanol/cyclohexane (red), cyclohexanol/n-hexane (green) and cyclohexanol/cyclohexane (magenta) mixtures.

Table 1, 1-hexanol $/ n$-hexane is the most ideal mixture, while cyclohexanol $/ n$-hexane mixture is at the opposite end of the scale. It is worth to mention that the same trends were obtained both from ATR-IR and NIR spectra. In the case of ATR-IR excess absorption spectra, it was possible to estimate the separate values of $\mathrm{ER}$ for the $\mathrm{CH}$ and $\mathrm{OH}$ vibrations. In most mixtures $\mathrm{ER}$ for the $\mathrm{OH}$ vibrations are four times higher as compared with those for the $\mathrm{CH}$ vibrations. This means that the $\mathrm{OH}-\mathrm{OH}$ interactions more significantly contribute to deviations from the ideal mixture as compared with the hydrophobic interactions of the alkyl parts. The values of ER from OH vibrations are similar for both mixtures of 1-hexanol, but significantly smaller than the analogous values for the mixtures of cyclohexanol. Hence, one can conclude that ER is related to the degree of association of the alcohol. It is also evident that values of $\mathrm{ER}$ for the $\mathrm{CH}$ vibrations are smaller if the chains have the same structure.

Comparison of ER for alcohol/alcohol mixtures (Table 2, ref. 16) with those for alcohol/alkane mixtures (Table 1, this work)

Table 1 Values of ER for all studied mixtures

\begin{tabular}{llllll}
\hline & \multicolumn{2}{l}{ ATR-IR } & & & \multicolumn{2}{l}{ NIR } \\
\cline { 2 - 3 } Mixture & $\mathrm{CH}+\mathrm{OH}$ & $\mathrm{CH}$ & $\mathrm{OH}$ & & $\mathrm{CH}+\mathrm{OH}$ \\
\hline 1-Hexanol $/ n$-hexane & 2.1 & 1.0 & 4.0 & 1.4 \\
1-Hexanol/cyclohexane & 3.5 & 2.7 & 4.9 & 2.8 \\
Cyclohexanol/cyclohexane & 3.8 & 1.8 & 7.4 & 2.4 \\
Cyclohexanol $/ n$-hexane & 9.9 & 4.7 & 19.1 & 4.2
\end{tabular}




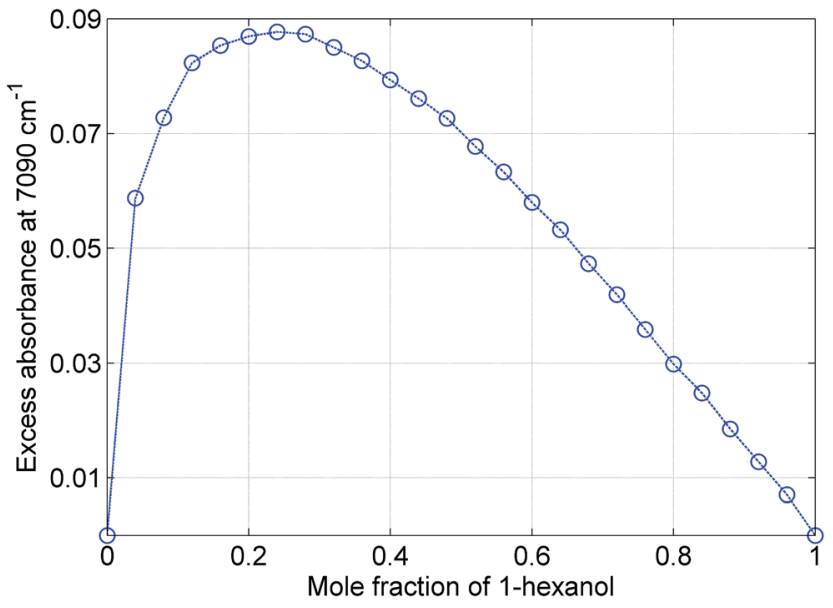

Fig. 6 The spectral changes at $7090 \mathrm{~cm}^{-1}$ in NIR excess absorption spectrum of 1 -hexanol $/ n$-hexane mixture.

reveals that the values from ATR-IR spectra are comparable. In contrast, values of ER obtained from NIR spectra are significantly higher for alcohol/alcohol mixtures than those for alcohol/alkane mixtures. An inspection of Fig. 11b (ref. 16) and Fig. 5b (this work) immediately explains the reason of the difference. In alcohol/alcohol mixtures the main contribution to ER comes from the bonded $\mathrm{OH}$. On the other hand, this band is much weaker in alcohol/alkane mixtures.

Fig. 6 displays the intensity variations at $7090 \mathrm{~cm}^{-1}$ in the NIR excess absorption spectrum of 1 -hexanol $/ n$-hexane mixture. Similar plots were obtained for the other mixtures (not shown). As can be seen, the largest deviation from the ideal mixture appears at $X_{\text {alc }} \approx 0.2-0.3$. It is of note that at this mole fraction is observed the highest population of the free $\mathrm{OH}$ groups (Fig. 4). This suggests that deviation from the ideality in alcohol/alkane mixtures is related to the population of the free $\mathrm{OH}$.
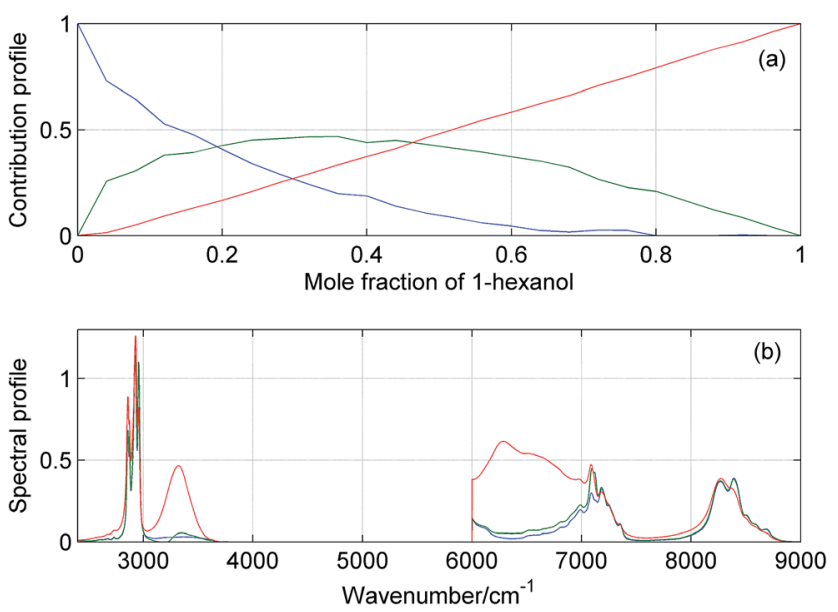

Fig. 7 Concentration (a) and spectral (b) profiles obtained from MCRALS of ATR-IR + NIR spectra of 1-hexanol/n-hexane mixture by using three components. Corresponding concentration and spectral profiles have the same colors.
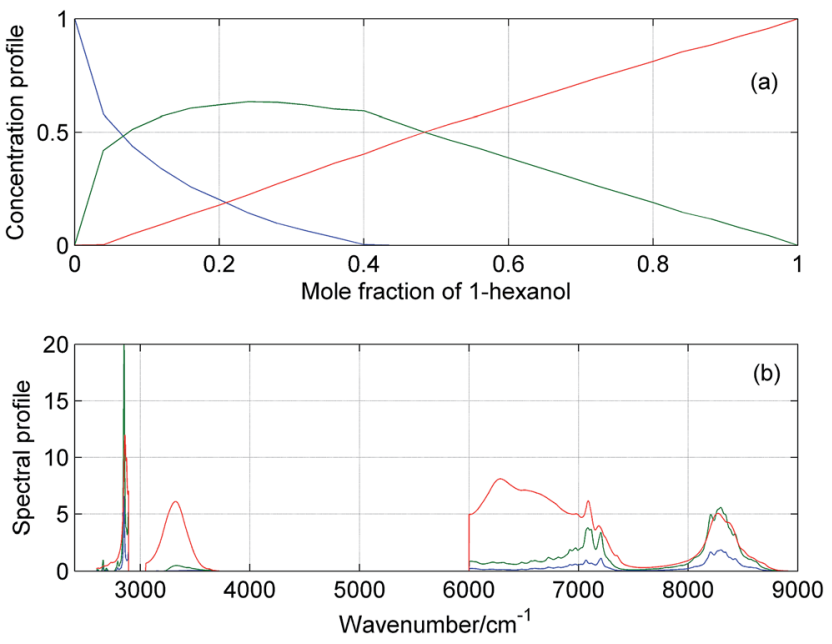

Fig. 8 Concentration (a) and spectral (b) profiles obtained from MCRALS of ATR-IR + NIR spectra of 1-hexanol/cyclohexane mixture by using three components. Corresponding concentration and spectral profiles have the same colors.

\section{Resolution of ATR-IR/NIR spectra of the mixtures by MCR-ALS}

Using the same computational procedures as previously, ${ }^{16}$ we estimated the number of significant components in the mixtures. In most mixtures the results suggested the presence of 2-3 components, only for cyclohexanol $/ n$-hexane mixture the best fits were obtained for 3-4 components. To increase the reliability of MCR-ALS results, we preformed the calculations by using the augmented data matrix, which included both ATR-IR and NIR spectra. The concentration and spectral profiles obtained from MCR-ALS are shown in Fig. 7-10. For cyclohexanol/ $n$-hexane mixture the best results were obtained by using four components, while the remaining three mixtures provided the best results with three components. The concentration profiles shown in Fig. 7a-9a are similar. The profile assigned to alkane rapidly decreases, while the alcohol's profile gradually increases with an increase in the alcohol content. The third profile ('green') after initial fast increase reaches the maximum near $X_{\text {alc }} \approx 0.3$ and then gradually decreases. The spectral profile of this component is similar to that of pure alkane, but additionally it includes a significant peak near $7100 \mathrm{~cm}^{-1}$ due to absorption of the free $\mathrm{OH}$. It is worth to note that this spectral component does not reveal the contribution from the associated $\mathrm{OH}$. An estimated (from the concentration profiles) alkane : alcohol ratio for this cluster was found to be higher than 8 10. This means that the third component is a heterocluster consisting of a single molecule of alcohol surrounded by the molecules of alkane. The molecule of alcohol in this cluster do not interact with the other molecules of alcohol, and for this reason the $\mathrm{OH}$ group absorb near $7100 \mathrm{~cm}^{-1}$.

Fig. 10 display results of MCR-ALS for cyclohexanol $/ n$-hexane mixture. As can be seen, the concentration profile of $n$-hexane is more or less similar to those for the other alkanes. In contrast, the concentration profile of cyclohexanol is different from analogous profiles of alcohols in the other mixtures. The formation of pure cyclohexanol clusters starts at $X_{\text {alc }} \approx 0.3$, 

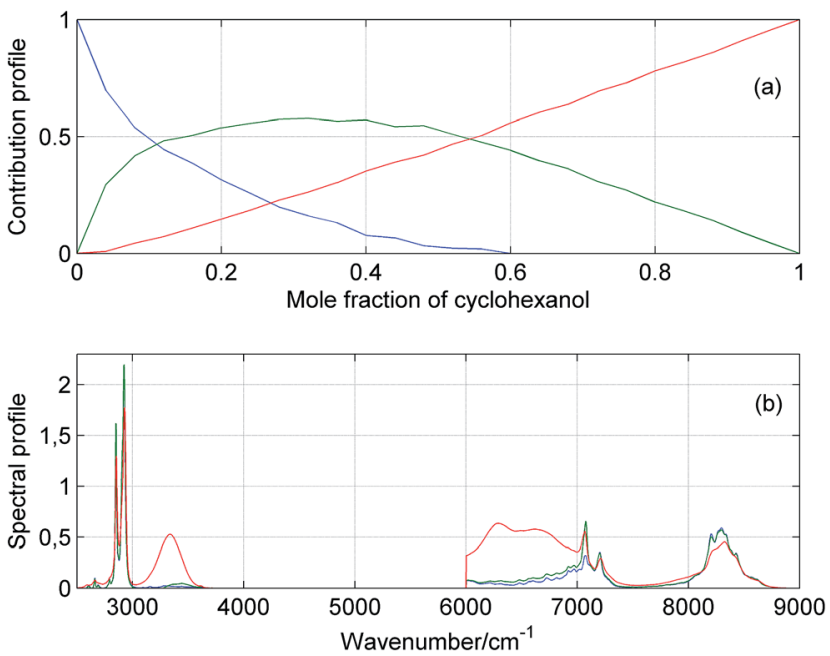

Fig. 9 Concentration (a) and spectral (b) profiles obtained from MCRALS of ATR-IR + NIR spectra of cyclohexanol/cyclohexane mixture by using three components. Corresponding concentration and spectral profiles have the same colors.

while in the remaining mixtures it starts at $X_{\text {alc }} \approx 0.04$. In addition, an increase in the population of cyclohexanol clusters is strongly nonlinear (Fig. 10a). The 'green' heterocluster is similar to those in the other mixtures, and it consists mainly from molecules of $n$-hexane. The molecules of cyclohexanol in these clusters do not interact with the other molecules of cyclohexanol. In contrast, the spectral profile of the second heterocluster ('red') is similar to that of the alcohol (Fig. 10b). The intensity ratio free : bonded $\mathrm{OH}$ for this spectral profile is only slightly higher than that for the pure cyclohexanol. Hence, one can conclude that these clusters are built mainly from the hydrogen-bonded molecules of cyclohexanol with a small
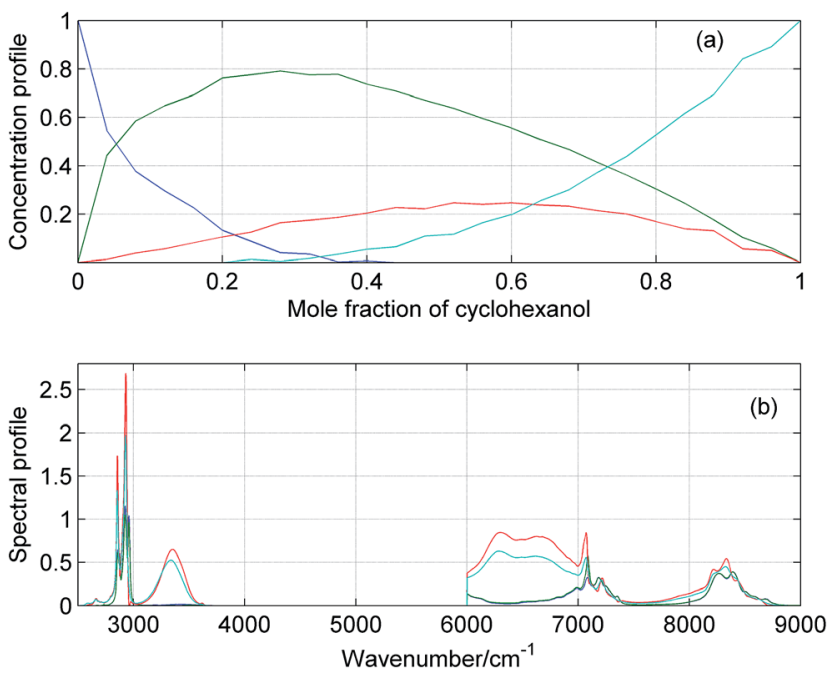

Fig. 10 Concentration (a) and spectral (b) profiles obtained from MCR-ALS of ATR-IR + NIR spectra of cyclohexanol/n-hexane mixture by using four components. Corresponding concentration and spectral profiles have the same colors.
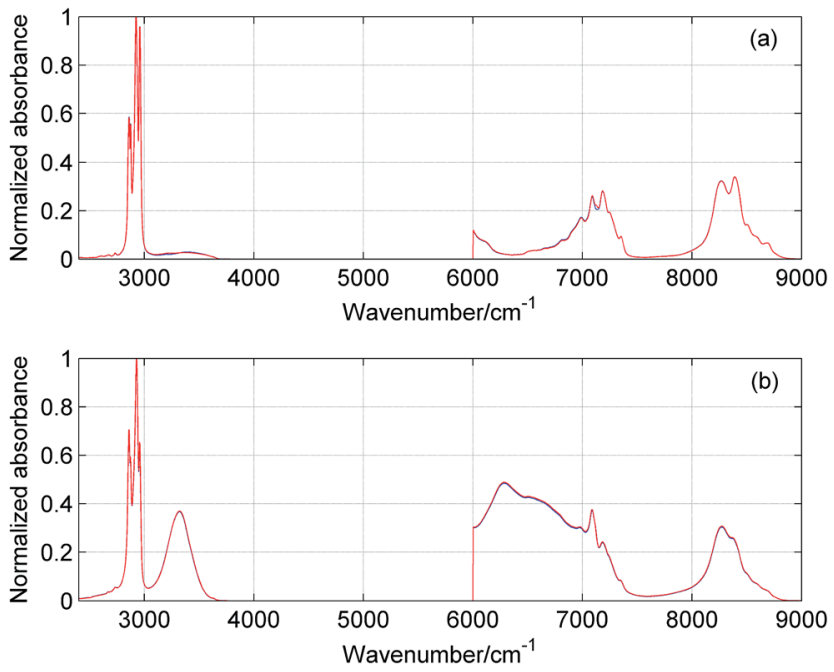

Fig. 11 Normalized (to unity) spectra (blue) of pure $n$-hexane (a) and 1-hexanol (b) together with the corresponding spectral profiles (red) obtained from MCR-ALS of ATR-IR + NIR spectra of 1-hexanol/nhexane mixture by using three components.

contamination of $n$-hexane. At maximum $\left(X_{\text {alc }} \approx 0.6\right)$ only $25 \%$ of molecules are involved in these alcohol-rich clusters.

In Fig. 11 are shown the normalized spectral profiles of 1hexanol (a) and $n$-hexane (b) in 1-hexanol $/ n$-hexane mixture together with the corresponding spectra of pure 1-hexanol and $n$-hexane. Similar plots were obtained for the remaining mixtures (not shown). It is clear that the spectral profiles of $n$ hexane (Fig. 11a) and 1-hexanol (Fig. 11b) are identical with the corresponding spectra of bulk $n$-hexane and 1-hexanol. This evidences that the homoclusters of alcohol and alkane in the mixture are similar to those in neat components. However, both kinds of homoclusters exist in a different range of compositions (Fig. 7a-9a).

\section{Conclusions}

It has been shown that in 1-hexanol/n-hexane, 1-hexanol/cyclohexane and cyclohexanol/cyclohexane mixtures the homoclusters of alcohols exist in the entire range of mole fractions, whereas the homoclusters of alkane are present only up to $X_{\text {alc }} \approx$ 0.4-0.6. Evidently, the clusters created by the $\mathrm{OH}-\mathrm{OH}$ interactions are more stable than those formed by the hydrophobic interactions of the alkyl chains. The alkane-rich heteroclusters exist in the entire range of compositions with maximum near $X_{\text {alc }} \approx 0.3$. At this mole fraction about $50-60 \%$ molecules are involved in the heteroclusters. Only in cyclohexanol $/ n$-hexane mixture this fraction is significantly higher - nearly $80 \%$ at maximum. These alkane-rich heteroclusters consist of a single molecule of alcohol surrounded by more than 8-10 molecules of alkane. In cyclohexanol $/ n$-hexane mixture we found an additional alcohol-rich cluster. This cluster exists in the entire range of mole fractions and has the maximum at $X_{\text {alc }} \approx 0.6$.

From MIR/NIR excess absorption spectra and values of ER it results that 1 -hexanol $/ n$-hexane mixture is the closest to the 
ideal mixture. In contrast, the highest deviation from the ideality appears for cyclohexanol $/ n$-hexane mixture. The maximum of these deviations, for all studied mixtures, occurs at $X_{\text {alc }} \approx 0.2-0.3$. As shown, the extent of deviation from the ideality in alcohol/alkane mixtures depends on the degree of association of the alcohol and the similarity of the alkyl parts. More associated is the alcohol and more similar are the alcohol and alkane chains, the more ideal is the mixture. As 1-hexanol is more associated than cyclohexanol, 1 -hexanol $/ n$-hexane mixture is the closest to the ideal mixture. In contrast, cyclohexanol $/ n$ hexane is the most non-ideal mixture since in this case the smaller extent of self-association of cyclohexanol is coupled with different structure of the alcohol and alkane chains. Our studies provide evidences that the $\mathrm{OH}-\mathrm{OH}$ interactions more significantly contribute to the deviation from the ideality as compared with the interactions of the alkyl parts.

\section{Acknowledgements}

The authors gratefully acknowledge Ewa Dłużniewska for the assistance in measuring spectra. This work was supported by Grant NCN 2013/11/B/ST4/00501.

\section{References}

1 A. N. Fletcher and C. A. Heller, J. Phys. Chem., 1967, 71, 3742.

2 N. Asprion, H. Hasse and G. Maurer, Fluid Phase Equilib., 2001, 186, 1.

3 M. Kunst, D. D. Duijn and P. Bordewijk, Ber. Bunsen-Ges. Phys. Chem., 1979, 83, 840.

4 M. Iwahashi, Y. Hayashi, N. Hachiya, H. Matsuzawa and H. Kobayashi, J. Chem. Soc., Faraday Trans., 1993, 89, 707.

5 J. M. Stubbs and J. I. Siepmann, J. Am. Chem. Soc., 2005, 127, 4722.

6 R. B. Gupta and R. L. Brinkley, AIChE J., 1998, 44, 207.
7 N. von Solms, L. Jensen, J. L. Kofod, M. L. Michelsen and G. M. Kontogeorgis, Fluid Phase Equilib., 2007, 261, 272.

8 L. Wilson, R. B. Alencastro and C. Sandorfy, Can. J. Chem., 1985, 63, 40.

9 M. A. Czarnecki, Appl. Spectrosc. Rev., 2011, 46, 67.

10 M. A. Czarnecki, Y. Morisawa, Y. Futami and Y. Ozaki, Chem. Rev., 2015, 115, 9707.

11 S. Schwerdtfeger, F. Köhler, R. Pottel and U. Kaatze, J. Chem. Phys., 2001, 115, 4186.

12 M. E. Flores, T. Shibue, N. Sugimura, H. Nishide, F. Martinez, A. F. Olea and I. Moreno-Villoslada, J. Mol. Liq., 2014, 199, 301.

13 J. J. Max and C. Chapados, J. Chem. Phys., 2008, 128, 224512. 14 J. T. Reilly, A. Thomas, A. R. Gibson, C. Y. Luebehusen and M. D. Donohue, Ind. Eng. Chem. Res., 2013, 52, 14456.

15 C. Mello, T. Mello, E. Sevéri, L. Coelho, D. Ribeiro, A. Marangoni, R. J. Poppi and I. Noda, J. Chem. Phys., 2009, 131, 084501.

16 W. Wrzeszcz, P. Tomza, M. Kwaśniewicz, S. Mazurek, R. Szostak and M. A. Czarnecki, RSC Adv., 2016, 6, 37195.

17 S. Wold, K. Esbensen and P. Geladi, Chemom. Intell. Lab. Syst., 1987, 2, 37.

18 H. R. Keller and D. L. Massart, Chemom. Intell. Lab. Syst., 1991, 12, 209.

19 R. Tauler, B. Kowalski and S. Fleming, Anal. Chem., 1993, 65, 2040.

20 R. Tauler, A. Izquierdo-Ridorsa and E. Casassas, Chemom. Intell. Lab. Syst., 1993, 18, 293.

21 Q. Li, N. Wang, Q. Zhou, S. Sun and Z. Yu, Appl. Spectrosc., 2008, 62, 166.

22 Y. Koga, F. Sebe, T. Minami, K. Otake, K. Saitow and K. Nishikawa, J. Phys. Chem. B, 2009, 113, 11928.

23 W. A. P. Luck and W. Ditter, J. Mol. Struct., 1967-1968, 1, 261.

24 R. Iwamoto, Appl. Spectrosc., 2009, 63, 354. 